\title{
Effect of gene polymorphisms and ethanol consumption on micronucleus frequency in human reticulocytes: a preliminary study
}

\author{
Chuancheng Wu $\cdot$ Yuquan Lu $\cdot$ Kanehisa Morimoto
}

Received: 22 October 2009/Accepted: 30 November 2009/Published online: 20 January 2010

(C) The Japanese Society for Hygiene 2010

\begin{abstract}
Objective Results from previous studies suggest that alcohol consumption can be genotoxic on peripheral lymphocytes. The aim of our study was to examine the association of alcohol consumption and its genotoxic effect on hematopoietic stem cells in vivo.

Methods We investigated 156 healthy Japanese males in a cross-sectional study. Lifestyles, including alcohol drinking behavior and cigarette smoking status, were investigated by means of a self-completed questionnaire. Polymorphisms of $A D H 1 B$ and $A L D H 2$ were identified by PCR-restriction fragment length polymorphism (RFLP) analysis. The presence of micronuclei in transferrin-positive reticulocytes (MN-RET) was detected with a single-laser flow cytometer. Associations between the genetic polymorphisms, lifestyle factors, and MN-RET frequency were statistically analyzed. Results We found a significant difference in the mean frequencies of MN-RET between habitual drinkers and non-habitual drinkers $(P=0.043)$, and between the $A L D H 2 * 1 / * 1$ and $A L D H 2 * 2 / * 2$ genotype $(P=0.015)$. The $A D H 1 B * 2$ and $A L D H 2 * 2$ haplotype was estimated to have a significantly higher influence on MN-RET frequency than the $A D H 1 B^{* 2}$ and $A L D H 2 * 1$ haplotype $(P=0.00035)$, and the frequency of alcohol consumption played a
\end{abstract}

C. Wu $\cdot$ Y. Lu $\cdot$ K. Morimoto $(\bowtie)$

Department of Social and Environmental Medicine,

Osaka University Graduate School of Medicine,

2-2 Yamada-oka, Suita, Osaka 565-0871, Japan

e-mail: morimoto@envi.med.osaka-u.ac.jp;

kanemorimoto@hotmail.com

K. Morimoto

Twin Research Center, Osaka University Graduate

School of Medicine, 2-2 Yamada-oka, Suita,

Osaka 565-0871, Japan significant role in MN-RET frequency on the background of the $A D H 1 B^{*} 2$ and $A L D H 2 * 1$ haplotype $(P=0.012)$. Conclusion The results of our study suggest a possible association between the $A D H 1 B$ and $A L D H 2$ polymorphism and the genotoxic effects of alcohol drinking on hematopoietic stem cells.

Keywords $A D H 1 B \cdot A L D H 2 \cdot$ Ethanol $\cdot$ Micronuclei · Reticulocyte

\section{Introduction}

Chromosome alterations, such as chromosomal aberrations (CAs), sister chromatid exchanges (SCEs), and micronuclei $(\mathrm{MN})$, which are directly visible as changes in chromosome structure, have been used as biomarkers for studying individuals exposed to known or potential genetic agents [1]. Among these biomarkers, MN appear to be the most suitable for epidemiological studies because it is simple and can reflect changes due to early-stage carcinogenesis. $\mathrm{MN}$ originate from chromosome fragments or whole chromosomes that are not included in the main daughter nuclei during nuclear division, and it has been suggested that MN plays an as yet unknown but important role in the occurrence of genetic instability and cancer [2].

The frequency of MN can also be measured in human reticulocytes as a means to evaluate cytogenetic damage to hematopoietic stem cells resulting from chemo- or radiotherapy or unhealthy lifestyle factors [3-5]. To date, these human reticulocyte studies have primarily used bone marrow aspirate or peripheral blood from splenectomized subjects (because human spleen can remove reticulocytes from the blood stream in 1 day, leaving only about $1 \%$ in the red blood cells). Abramsson-Zetterberg et al. [6] 
described a method for measuring MN in enriched transferrin-positive reticulocytes (trf-ret). They found that $\mathrm{MN}$ frequency in reticulocytes approximated those observed in bone marrow in mice and suggested that scoring young trfret for $\mathrm{MN}$ is a good substitute for the method using inaccessible bone marrow [7].

Several enzymes are involved in the metabolic transformation of ethanol. In the major pathway, alcohol dehydrogenase $(\mathrm{ADH})$ metabolizes most ethanol in the cytoplasm of the liver cells, and the intermediary metabolite, acetaldehyde, is subsequently oxidized to acetate predominantly by aldehyde dehydrogenase-2 (ALDH2), which is mainly located in hepatic mitochondria $[8,9]$.

A single nucleotide polymorphism (SNP) in $A D H 1 B$ is rs1229984, which changes an arginine to a histidine at residue 47. This change enhances the alcohol-oxidizing activity of $A D H 1 B$, with the homodimers of the mutant allele $A D H 1 B^{*} 2$ (resulting from 47Arg $\rightarrow$ His in exon 3 ) showing an approximately 40-fold higher alcohol-oxidizing activity than the heterodimers $A D H 1 B^{*} 1 / * 2$ and an approximately 100 -fold higher activity than the $A D H 1 B^{*} 1$ homodimers $[10$, 11]. The $A D H 1 B * 2$ allele is common in Asian populations (the frequency ranges from 10 to $90 \%$ ), but less common in African or European populations (15 or $10 \%$, respectively) $[12,13]$. ADH1B* $1 / * 1$ is genetically recessive to the mutant.

The $A L D H 2$ polymorphism results from the substitution of a glutamate for a lysine at residue 487 . The alleles that encode the active and inactive subunits are $A L D H 2 * 1$ and $A L D H 2 * 2$, respectively. Individuals with the $A L D H 2 * 1 / * 2$ heterozygote have been estimated to have approximately $6 \%$ of the enzymatic activity of those with the wild-type $A L D H 2$, while those with the $A L D H 2 * 2 / * 2$ genotype have been estimated to have lost almost all of their $A L D H 2$ activity. Thus, both the $A L D H 2 * 1 / * 2$ heterozygote and $A L D H 2 * 2 / * 2$ homozygote are usually characterized as an ALDH2-deficient phenotype [14]. Apparently, the active ALDH2 allele is recessive to the mutant. Frequency of $A L D H 2 * 2$ mutant allele in Asia is reportedly is about $40 \%$, whereas less than 5\% among both European and African populations [12].

Individuals with the $A L D H 2 * 2 / * 2$ genotype are reported to have a higher serum acetaldehyde level among alcohol drinkers [15]. A large number of studies have shown that $A D H 1 B$ and $A L D H 2$ polymorphisms have a significant association with the alcohol-related genotoxicity in peripheral blood lymphocytes. However, relatively little population data are available to illustrate the genotoxic effects of lifestyle and genetic factors on the bone-marrow hematopoietic stem cells. The aim of this study was to investigate the association of the $A D H 1 B$ and $A L D H 2$ polymorphisms and alcohol drinking behavior with the frequencies of $\mathrm{MN}$ in reticulocytes among Japanese male subjects.
Our study cohort consisted of the employees of a hardmetal tooling factory in Osaka. These employees were receiving annual physical examinations within the framework of a longitudinal study we are carrying out on lifestyle factors. As the number of female employees in this factory is relatively small, and women have different lifestyles from men, only male employees were enrolled in the study to exclude possible confounding effects from gender.

\section{Materials and methods}

Subjects and blood samples

This study was approved by the Ethics Committee at Osaka University Medical School. Informed consent was asked and received for enrollment in this cross-sectional study. To reduce the possibility of confounding factors, we recruited 156 daily male workers from a hard-metal tooling factory in Osaka, Japan in 2007 and asked each participant to answer a self-completed questionnaire on his daily lifestyle, including age, cigarette smoking, alcohol drinking, physical activity, nutritional balance, and occupation (official staff and technical work or manufacturing work), among other things (Table 1). All of the questionnaires were anonymous, and each questionnaire carried a code number. Information on the lifestyle data of the donors was not made known before the measurement of micronuclei in transferrin-positive reticulocytes (MN-RET) was completed.

\section{Assay for MN-RET}

The assay for MN-RET was carried out as described by Offer et al. [16].

\section{Enrichment of trf-ret}

A certified phlebotomist collected blood samples using coded 5-ml EDTA vacutainer tubes through venipuncture. The fresh red blood cell (RBC) fraction ( $2 \mathrm{ml}$ ) was separated from whole blood by centrifugation and the plasma and buffy-coat layer discarded. The RBC fraction was incubated with BioMag Anti-CD71 beads and treated with a MPC-S magnet (Dynal Biotech, Oslo, Norway) for $15 \mathrm{~min}$ at room temperature. Unbound cells were aspirated, and the bound cells (enriched trf-ret) were washed twice, suspended in $200 \mu \mathrm{lBS} / 5 \%$ fetal bovine serum (FBS), and transferred to a new tube. The enriched trf-ret was labeled with CD71-FITC antibody by incubation for $30 \mathrm{~min}$ in the dark. The samples were then centrifuged, resuspended in $100 \mu \mathrm{l}$ heparinized HBS, and fixed by forceful pipetting into $1 \mathrm{ml}$ ultracold $\left(-80^{\circ} \mathrm{C}\right)$ methanol. The fixed samples were stored at $-80^{\circ} \mathrm{C}$ until use [16]. 
Table 1 Questionnaire for investigation of lifestyles among the study subjects

\begin{tabular}{|c|c|c|c|c|c|}
\hline 1. Age & ( ) years & & & & \\
\hline 2. Occupation & (1) Manufacturing etc. & (2) Official etc. & & & \\
\hline 3. Education & (1) Middle school & (2) High school & (3) Technical college & (4) University or higher & \\
\hline 4. Sports & (1) Almost everyday & (2) 2-4 times/week & (3) 1 time/week & (4) 1 time/month & (5) Almost none \\
\hline 5. Alcohol & (1) Almost everyday & (2) 3-5 times/week & (3) 1-2 times/week & (4) $1-3$ times/month & (5) $<1$ time/month \\
\hline 6. Cigarette & (1) Smoker & (2) Former smoker & (3) Never smoked & & \\
\hline 7. Sleeping hours (h) & (1) $\geq 9$ & (2) 8 & (3) 7 & (4) 6 & $(5) \leq 5$ \\
\hline 8. Nutrition balance & (1) Pays attention & (2) Pays little attention & (3) Pays no attention & & \\
\hline 9. Breakfast & (1) Almost everyday & (2) Sometimes & (3) Rarely & & \\
\hline 10. Working hours (h) & (1) $\geq 11$ & (2) 10 & (3) 9 & (4) 8 & (5) $\leq 7$ \\
\hline 11. Mental stress & (1) Much & (2) General & (3) Less & & \\
\hline
\end{tabular}

\section{Staining of micronuclei}

We resuspended $0.5 \mathrm{ml}$ of the fixed reticulates in $2.5 \mathrm{ml}$ HBSS buffer, following which the samples were first mixed and then centrifuged. Supernatants were aspirated, and cells were washed twice before being suspended in $2 \mathrm{ml}$ HBSS buffer. The suspended cells was transferred to a FACS tube, FBS solution and RNase were added to the cells in the FACS tube, and the suspension was incubated for $30 \mathrm{~min}$ in the dark to remove RNA from the cells. The cells were then mixed with HBS solution containing 7-aminoactinomycin (7AAD) and incubated for another $30 \mathrm{~min}$ in the dark. These cells were then used for detection of $\mathrm{MN}$ with a flow cytometer. The position of the quadrant used to distinguish between trf-ret and mature erythrocytes (RBCs) was set by running a control unlabeled with CD71.

\section{Flow cytometry}

Samples were analyzed on an argon ion laser FACSort device (Becton-Dickinson, Sunnyvale, CA) equipped with standard optics. A live gate was used in the forward/side scatter parameters to exclude debris and residual nucleated cells, thereby restricting data acquisition primarily to the trfret subset. Data from approximately 200,000 trf-ret were acquired. CellQuest software was used for data acquisition and analysis. In the analysis, quadrant plot regions of interest were defined for RBCs, trf-ret, and micronucleated trf-ret (MN-RET). The number of events in each region was determined, and the relative frequencies were calculated from the number of events in the upper-right quadrant (MNRET region) divided by the total number of events in the upper-quadrant plot regions (trf-ret region).

$A D H 1 B$ and $A L D H 2$ genotype analysis

DNA was extracted from whole blood samples. The $A D H 1 B$ genotype at codon 48 and the $A L D H 2$ polymorphism at codon 487 were determined by PCRrestriction fragment length polymorphism (PCR-RFLP) analysis [17]. In brief, the genomic DNA sample was amplified using two primers for the $A D H 1 B$ polymorphism fragment [5'-AAT CTT TTC TGA ATC TGA ACA G-3' (forward) and 5'-GAA GGG GGG TCA CCA GGT TGC$3^{\prime}$ (reverse)] and two primers for the $A L D H 2$ polymorphism fragment [5'-CAA ATT ACA GGG TCA ACT GCT-3' (forward) and 5'-CCA CAC TCA CAG TTT TCT CTT-3' (reverse)]. The PCR products were treated with restriction enzymes (MaeIII for $A D H 1 B$ and $M b o$ II for $A L D H 2$ ). The DNA-digested samples for the two genes were then electrophoresed on $3 \%$ agarose and $12 \%$ polyacrylamide gels.

\section{Statistical analysis}

We applied the Student's $t$-test and one-way analysis of variance (ANOVA) for statistical analysis of the data using SPSS ver. 15.0 for Windows (SPSS, Chicago, IL). The Kolmogorov-Smirnov test was used to test the normal distributions. The statistical power was calculated using G*Power software (HHU, Gebäude, Germany). Thesias ver. 3.1 software [18] was used to calculate quantitative trait loci (QTL) phenotypes as dependent variables, with covariates of genotype, age, cigarette smoking, or alcohol drinking behaviors, among others.

\section{Results}

The mean age of the subjects was 45.2 years (range 22 64 years). As shown in Table 2, the subjects who aged $>45$ years had a significantly higher mean MN-RET frequency than those who were $\leq 45$ years of age $(P=0.043)$ at a statistical power of 0.52 . Subjects who were habitual drinkers ( $\geq 3$ times a week) showed a significantly higher mean MN-RET frequency than non-habitual drinkers $(<3$ times a week $(P=0.015)$ at a statistical power of 0.68 . 
Table 2 Mean micronuclei in transferrin-positive reticulocyte frequency by age, drinking frequency, and smoking status

\begin{tabular}{|c|c|c|}
\hline Covariates & $\begin{array}{l}\text { Number } \\
\text { of subjects }\end{array}$ & $\% \mathrm{MN}-\mathrm{RET}$ \\
\hline \multicolumn{3}{|l|}{ Age } \\
\hline Younger subjects ( $\leq 45$ years) & 69 & $0.050 \pm 0.0051$ \\
\hline Elderly subjects ( $>45$ years) & 87 & $\begin{array}{l}0.064 \pm 0.0048 * \\
\quad(0.52)^{\mathrm{a}}\end{array}$ \\
\hline \multicolumn{3}{|l|}{ Drinking frequency } \\
\hline $\begin{array}{l}\text { Habitual drinkers } \\
\text { ( } \geq 3 \text { times a week) }\end{array}$ & 74 & $0.067 \pm 0.0052$ \\
\hline $\begin{array}{l}\text { Non-habitual drinkers } \\
(<3 \text { times a week })\end{array}$ & 82 & $\begin{array}{l}0.050 \pm 0.0046^{*} \\
(0.68)^{\mathrm{a}}\end{array}$ \\
\hline \multicolumn{3}{|l|}{ Smoking status } \\
\hline Never & 64 & $0.052 \pm 0.0050$ \\
\hline Ever $^{\mathrm{b}}$ & 92 & $0.062 \pm 0.0048$ \\
\hline
\end{tabular}

* $P<0.05$ (Student's $t$-test)

a $\% \mathrm{MN}-\mathrm{RET}$ is the frequency of micronuclei in transferrin-positive reticulocytes (MN-RET), expressed as the mean \pm standard error (SE), with the statistical power given in parenthesis

b Including former smokers

No significant difference in the MN-RET frequency was observed between the smoking and non-smoking subgroups $(P=0.153)$, and the other lifestyle factors also did not affect MN-RET frequency (data not shown).

Table 3 summarizes the distribution of all subjects among the $A D H 1 B$ and $A L D H 2$ genotypes and the mean MN-RET frequency for each genotypic subgroup. The genotypic frequencies for the two polymorphisms were calculated and found to be in Hardy-Weinberg equilibrium. MN-RET frequency was found to be differentially distributed among the subjects carrying the $A L D H 2 * 1 / * 1$, $A L D H 2 * 1 / * 2$, and $A L D H 2 * 2 / * 2$ genotypes $(P=0.047)$ at a statistical power of 0.332 , but it was not significantly differentially distributed among $A D H 1 B^{*} 1 / * 1, A D H 1 B^{*} 1 / * 2$, and $A D H 1 B^{*} 2 / * 2$ genotypes $(P=0.071)$ at a statistical power of 0.497 .

The effects of the $A D H 1 B$ and $A L D H 2$ haplotype on MN-RET frequency were estimated using Thesias software. The results showed that from among all of the different combinations of $A D H 1 B$ and $A L D H 2$ haplotypes, the only significant difference in MN-RET frequency was between the $A D H 1 B^{*} 2-A L D H 2 * 2$ haplotype combination and the most frequent haplotype combination, $A D H 1 B^{*} 2-$ $A L D H 2 * 1(P=0.00035)$ (Table 4).

The influence of age, cigarette smoking, and alcohol drinking on the MN-RET frequency under the genetic background of the $A D H 1 B^{*} 2-A L D H 2 * 2$ haplotype was estimated. The results showed that alcohol drinking was significantly associated with MN-RET frequency $(P=0.012)$. However, age and cigarette smoking failed to show a similar influence $(P=0.823$ and $P=0.383$,
Table 3 Mean \%MN-RET frequencies among $A D H 1 B$ and $A L D H 2$ genotypes

\begin{tabular}{lll}
\hline Genotype & Number of subjects $(\%)$ & $\%$ MN-RET $^{\mathrm{a}}$ \\
\hline$A D H 1 B$ & & \\
$* 1 / * 1$ & $13(8.3)$ & $0.043 \pm 0.0096$ \\
$* 1 / * 2$ & $60(38.5)$ & $0.051 \pm 0.0053$ \\
$* 2 / * 2$ & $83(53.2)$ & $0.065 \pm 0.0051$ \\
& $P$ for $\mathrm{HW}^{\mathrm{b}}=0.645$ & $P=0.071^{\mathrm{c}}(0.497)$ \\
$A L D H 2$ & & \\
$* 1 / * 1$ & $83(53.2)$ & $0.055 \pm 0.0039$ \\
$* 1 / * 2$ & $59(37.8)$ & $0.058 \pm 0.0064$ \\
$* 2 / * 2$ & $14(9.0)$ & $0.080 \pm 0.016$ \\
& $P$ for $\mathrm{HW}^{\mathrm{b}}=0.456$ & $P=0.047^{\mathrm{c}}(0.332)$
\end{tabular}

${ }^{a}$ Mean \pm standard error (SE), with the statistical power given in parenthesis

b $P$ value for deviation from Hardy-Weinberg equilibrium

${ }^{c} P$ for one-way ANOVA (analysis of variance)

Table 4 MN-RET frequency according to $A D H 1 B$ and $A L D H 2$ haplotypes

\begin{tabular}{|c|c|c|c|c|}
\hline \multicolumn{2}{|c|}{ Haplotype } & \multirow[t]{2}{*}{ Frequency } & \multirow{2}{*}{$\begin{array}{l}\text { Differences in MN-RET } \\
\text { frequency }(95 \% \mathrm{CI})\end{array}$} & \multirow[t]{2}{*}{$P$} \\
\hline$A D H 1 B$ & $A L D H 2$ & & & \\
\hline 2 & 1 & $0.539^{\mathrm{a}}$ & 0 & \\
\hline 2 & 2 & 0.185 & $0.0222(0.0100$ to 0.0343$)$ & 0.00035 \\
\hline 1 & 1 & 0.182 & $0.00063(-0.0148$ to 0.0160$)$ & 0.936 \\
\hline 1 & 2 & 0.092 & $-0.0119(0.0316$ to 0.0079$)$ & 0.239 \\
\hline
\end{tabular}

a The highest haplotype frequency as the control

Table 5 Covariable effects of age, alcohol drinking, cigarette smoking and $A D H 1 B * 2-A L D H 2 * 2$ haplotype on MN-RET frequency

\begin{tabular}{lll}
\hline Factors & $\begin{array}{l}\text { Differences in MN-RET } \\
\text { frequency }(95 \% \mathrm{CI})\end{array}$ & $P$ \\
\hline $\begin{array}{l}A D H 1 B^{*} 2- \\
A L D H 2^{*} 2^{\mathrm{a}} \times \text { age }\end{array}$ & $0.00270(-0.0210$ to 0.0265$)$ & 0.823 \\
$\begin{array}{l}A D H 1 B^{*} 2- \\
A L D H 2^{*} 2 \times \text { alcohol }\end{array}$ & $0.03434(0.00748$ to 0.0612$)$ & 0.012 \\
$\begin{array}{l}\text { drinking } \\
A D H 1 B^{*} 2-\end{array}$ & $0.01198(-0.0149$ to 0.03890$)$ & 0.383 \\
$\begin{array}{l}A L D H 2^{*} 2 \times \text { cigarette } \\
\text { smoking }\end{array}$ & & \\
\hline
\end{tabular}

CI Confidence interval

a $A D H 1 B^{* 2}-A L D H 2 * 2$ haplotype

respectively) (Table 5). We also examined the associations of occupation, education, physical activities, nutritional balance, and working hours with MN-RET frequency and found no significant relationship (data not shown). 


\section{Discussion}

Earlier studies have shown that alcohol drinking can affect bone marrow cells in human populations [19]. Wickramasinghe et al. [20] suggested the possibility that ethanol is metabolized in some human bone marrow cells, and Latvala et al. [21] suggested that acetaldehyde may be formed in bone marrow from alcohol and that the acetaldehyde-erythrocytes adducts would play a role in the generation of erythrocyte abnormalities.

Meagher et al. [22] reported that both ethanol (0.05$0.2 \%)$ and acetaldehyde $(0.001 \%)$ are able to suppress the proliferation of hematopoietic progenitor cells in in vitro mice and human culture systems. In an in vivo animal experiment, an intraperitoneal injection of acetaldehyde $(0.5 \mathrm{mg} / \mathrm{kg})$ induced a significant increase in SCEs in bonemarrow cells, while alcohol (10\% as the only liquid supplied for 46 weeks) failed to induce a similar genotoxicity [23]. These data demonstrate that acetaldehyde may play an important role in the genotoxicity of alcohol drinking in human populations or experimental animals and suggest that the efficiency of an individual's alcohol metabolism is an important determinant factor of the genotoxicity of alcohol drinking behaviors on marrow cells.

In our study, the $A D H 1 B * 2-A L D H 2 * 2$ haplotype showed a significantly higher MN-RET than the $A D H 1 B^{*} 2-A L D H 2 * 1$ haplotype. Further analysis showed that the frequency of alcohol consumption significantly interacted with MN-RET frequencies of the $A D H 1 B^{* 2}-$ $A L D H 2 * 2$ haplotype. These results indicate that alcohol drinking is associated with chromosome damage during the differentiation of erythrocytes in the bone marrow-under the modulation of genetic factors. To the best of our knowledge, this is the first report relating the effects of the combination of alcohol drinking and the $A D H 1 B$ and $A L D H 2$ polymorphisms on the frequency of micronuclei in reticulocytes isolated from fresh human peripheral blood.

We also observed that age was positively correlated with $\mathrm{MN}$ frequency in the reticulocytes at a lower statistical power, which is in line with results reported from a number of previous studies using lymphocytes [24, 25]. Nevertheless, the effect of age in our study decreased to nonsignificance under the background of the $A D H 1 B^{* 2}-$ $A L D H 2 * 2$ haplotype.

Cigarette smoking also failed to show a significant effect on MN-RET frequency in our study. In fact, although cigarette smoke contains many carcinogens and mutagens, the studies published to date that link smoking habits to MN frequency are rather controversial, with some showing a positive effect [26-28] and others showing no significance or even a negative effect [29-31]. The mechanisms underlying this phenomenon need to be studied in more detail.
No significant association between other factors, including occupation, working hours, physical activity, and nutritional balance, either singly or combined, and the $A D H 1 B-A L D H 2$ haplotype was found.

Alcohol drinkers with $A L D H 2$-deficient genotype are known to be able to accumulate acetaldehyde in the body easier than some other genotypes and, consequently, to suffer from its genotoxic damage [32]. Alcoholics have been found to have a higher frequency of SCEs, CAs, and $\mathrm{MN}$ in their lymphocytes than non-alcoholics [33-35], which is in line with the results of our study, with some slight differences. The MN observed in the peripheral blood lymphocytes reflect a complex exposure environment, including bone marrow, blood stream, cell cultures, or mutagen challenge in vitro. Lymphocytes themselves have undergone differentiation in the bone marrow, dormancy in the blood, and division in an in vitro environment. Thus, the MN detected in lymphocytes may result from an accumulative exposure to many environmental and genetic factors. In contrast, the MN-RET simply reflects the influence of bone-marrow environmental and genetic factors on the differentiating process of erythrocytes [36]. As such, it is a simpler and more direct biomarker for monitoring the genotoxicities of risk factors on the process of cell differentiation in vivo, i.e. on hematopoietic stem cells.

\section{Conclusion}

We have found an association among alcohol drinking frequency, $A D H 1 B$ and $A L D H 2$ polymorphisms, and $\mathrm{MN}$ RET in a group of human subjects. However, these results are only preliminary due to the small sample size and they need to be confirmed in future studies in a larger population.

Acknowledgments This work was partially supported by a Grantin-Aid for Scientific Research from the Japan Society for the Promotion of Science (JSPS) in 2007.

\section{References}

1. Norppa H. Cytogenetic markers of susceptibility: influence of polymorphic carcinogen-metabolizing enzymes. Environ Health Perspect. 1997;105[Suppl 4]:829-35.

2. Iarmarcovai $\mathrm{G}$, et al. Micronuclei frequency in peripheral blood lymphocytes of cancer patients: a meta-analysis. Mutat Res. 2008;659(3):274-83.

3. Goetz R, Sram RJ, Dohnalova J. Relationship between experimental results in mammals and man. I. Cytogenetic analysis of bone marrow injury induced by a single dose of cyclophosphamide. Mutat Res. 1975;31(4):247-54.

4. Jensen MK, Nyfors A. Cytogenetic effects of methotrexate on human cells in vivo: comparison between results obtained by chromosome studies on bone-marrow cells and blood 
lymphocytes and by the micronucleus test. Mutat Res. 1979;64(5):339-43.

5. Schlegel R, MacGregor JT, Everson RB. Assessment of cytogenetic damage by quantitation of micronuclei in human peripheral blood erythrocytes. Cancer Res. 1986;46(7):3717-21.

6. Abramsson-Zetterberg L, et al. Human cytogenetic biomonitoring using flow-cytometric analysis of micronuclei in transferrinpositive immature peripheral blood reticulocytes. Environ Mol Mutagen. 2000;36(1):22-31.

7. Abramsson-Zetterberg L, Grawe J, Zetterberg G. The micronucleus test in rat erythrocytes from bone marrow, spleen and peripheral blood: the response to low doses of ionizing radiation, cyclophosphamide and vincristine determined by flow cytometry. Mutat Res. 1999;423(1-2):113-24.

8. Badger TM, et al. Alcohol metabolism: role in toxicity and carcinogenesis. Alcohol Clin Exp Res. 2003;27(2):336-47.

9. Schoedel KA, Tyndale RF. Induction of nicotine-metabolizing CYP2B1 by ethanol and ethanol-metabolizing CYP2E1 by nicotine: summary and implications. Biochim Biophys Acta. 2003;1619(3):283-90.

10. Bosron WF, Li TK. Genetic polymorphism of human liver alcohol and aldehyde dehydrogenases, and their relationship to alcohol metabolism and alcoholism. Hepatology. 1986;6(3):50210 .

11. Bosron WF, Magnes LJ, Li TK. Kinetic and electrophoretic properties of native and recombined isoenzymes of human liver alcohol dehydrogenase. Biochemistry. 1983;22(8):1852-7.

12. Brennan $\mathrm{P}$, et al. Pooled analysis of alcohol dehydrogenase genotypes and head and neck cancer: a HuGE review. Am J Epidemiol. 2004;159(1):1-16.

13. Quertemont E. Genetic polymorphism in ethanol metabolism: acetaldehyde contribution to alcohol abuse and alcoholism. Mol Psychiatry. 2004;9(6):570-81.

14. Crabb DW, et al. Genotypes for aldehyde dehydrogenase deficiency and alcohol sensitivity. The inactive ALDH2(2) allele is dominant. J Clin Invest. 1989;83(1):314-6.

15. Neumark YD, et al. Alcohol dehydrogenase polymorphisms influence alcohol-elimination rates in a male Jewish population. Alcohol Clin Exp Res. 2004;28(1):10-4.

16. Offer $\mathrm{T}$, et al. A simple assay for frequency of chromosome breaks and loss (micronuclei) by flow cytometry of human reticulocytes. FASEB J. 2005;19(3):485-7.

17. Lee $\mathrm{CH}$, et al. Independent and combined effects of alcohol intake, tobacco smoking and betel quid chewing on the risk of esophageal cancer in Taiwan. Int J Cancer. 2005;113(3):475-82.

18. Tregouet DA, Garelle V. A new JAVA interface implementation of THESIAS: testing haplotype effects in association studies. Bioinformatics. 2007;23(8):1038-9.

19. Latvala J, Parkkila S, Niemela O. Excess alcohol consumption is common in patients with cytopenia: studies in blood and bone marrow cells. Alcohol Clin Exp Res. 2004;28(4):619-24.
20. Wickramasinghe SN, et al. Metabolism of ethanol by human bone marrow cells. Acta Haematol. 1981;66(4):238-43.

21. Latvala $\mathbf{J}$, et al. Acetaldehyde adducts in blood and bone marrow of patients with ethanol-induced erythrocyte abnormalities. Mol Med. 2001;7(6):401-5.

22. Meagher RC, Sieber F, Spivak JL. Suppression of hematopoieticprogenitor-cell proliferation by ethanol and acetaldehyde. N Engl J Med. 1982;307(14):845-9.

23. Korte A, et al. Influence of chronic ethanol uptake and acute acetaldehyde treatment on the chromosomes of bone-marrow cells and peripheral lymphocytes of Chinese hamsters. Mutat Res. 1981;88(4):389-95.

24. Barale R, et al. Sister chromatid exchange and micronucleus frequency in human lymphocytes of 1, 650 subjects in an Italian population: II. Contribution of sex, age, and lifestyle. Environ Mol Mutagen. 1998;31(3):228-42.

25. Fenech M, Rinaldi J. The relationship between micronuclei in human lymphocytes and plasma levels of vitamin $\mathrm{C}$, vitamin $\mathrm{E}$, vitamin B12 and folic acid. Carcinogenesis. 1994;15(7):1405-11.

26. $\mathrm{Au} \mathrm{WW}$, et al. Factors contributing to chromosome damage in lymphocytes of cigarette smokers. Mutat Res. 1991;260(2):137-44.

27. Tomanin R, et al. Influence of smoking habit on the frequency of micronuclei in human lymphocytes by the cytokinesis block method. Mutagenesis. 1991;6(2):123-6.

28. Vaglenov A, Karadjo A. Micronucleus frequencies in Bulgarian control populations. Cent Eur J Occup Environ Med. 1997;3:18794.

29. Bolognesi $\mathrm{C}$, et al. Cytogenetic biomonitoring in traffic police workers: micronucleus test in peripheral blood lymphocytes. Environ Mol Mutagen. 1997;30(4):396-402.

30. Bolognesi C, et al. Cytogenetic analysis of a human population occupationally exposed to pesticides. Mutat Res. 1993; 285(2):239-49.

31. Bukvic N, et al. Sister chromatid exchange (SCE) and micronucleus (MN) frequencies in lymphocytes of gasoline station attendants. Mutat Res. 1998;415(1-2):25-33.

32. Singh NP, Khan A. Acetaldehyde: genotoxicity and cytotoxicity in human lymphocytes. Mutat Res. 1995;337(1):9-17.

33. Butler MG, Sanger WG, Veonett GE. Increased frequency of sister-chromatid exchanges in alcoholics. Mutat Res. 1981; 85(2):71-6

34. Maffei F, et al. Increased cytogenetic damage detected by FISH analysis on micronuclei in peripheral lymphocytes from alcoholics. Mutagenesis. 2000;15(6):517-23.

35. Morimoto $\mathrm{K}$, Takeshita $\mathrm{T}$. Low $\mathrm{Km}$ aldehyde dehydrogenase (ALDH2) polymorphism, alcohol-drinking behavior, and chromosome alterations in peripheral lymphocytes. Environ Health Perspect. 1996;104[Suppl 3]:563-7.

36. Abramsson-Zetterberg L, Zetterberg G, Grawe J. The time-course of micronucleated polychromatic erythrocytes in mouse bone marrow and peripheral blood. Mutat Res. 1996;350(2):349-58. 